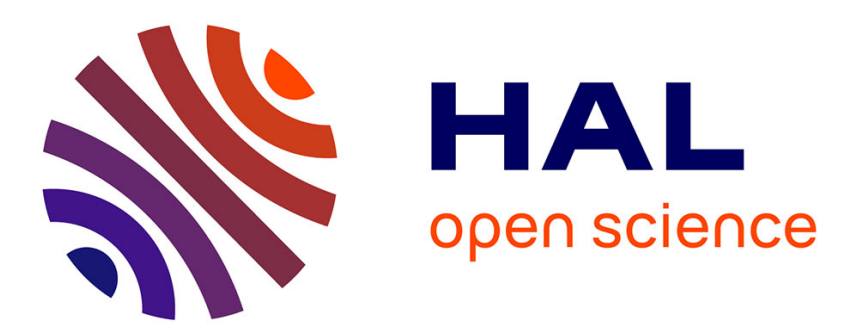

\title{
Heterogeneous Clutter Model for High-Resolution Polarimetric SAR Data Processing
}

Gabriel Vasile, Frédéric Pascal, Jean-Philippe Ovarlez, Pierre Formont

\section{To cite this version:}

Gabriel Vasile, Frédéric Pascal, Jean-Philippe Ovarlez, Pierre Formont. Heterogeneous Clutter Model for High-Resolution Polarimetric SAR Data Processing. IGARSS 2011 - IEEE International Geoscience and Remote Sensing Symposium, Jul 2011, Vancouver, Canada. pp.3744-3747, 10.1109/IGARSS.2011.6050039 . hal-00638874

\section{HAL Id: hal-00638874 https://hal.science/hal-00638874}

Submitted on 7 Nov 2011

HAL is a multi-disciplinary open access archive for the deposit and dissemination of scientific research documents, whether they are published or not. The documents may come from teaching and research institutions in France or abroad, or from public or private research centers.
L'archive ouverte pluridisciplinaire HAL, est destinée au dépôt et à la diffusion de documents scientifiques de niveau recherche, publiés ou non, émanant des établissements d'enseignement et de recherche français ou étrangers, des laboratoires publics ou privés. 


\title{
HETEROGENEOUS CLUTTER MODEL FOR HIGH RESOLUTION POLARIMETRIC SAR DATA PROCESSING
}

\author{
G. Vasile ${ }^{1}$, F. Pascal ${ }^{2}$, J. -P. Ovarlez ${ }^{2,3}$ and P. Formont ${ }^{2}$ \\ ${ }^{1}$ : Grenoble-Image-sPeach-Signal-Automatics Lab, CNRS / Grenoble-INP, FRANCE, gabriel.vasile@gipsa-lab.grenoble-inp.fr \\ 2 : SONDRA, Supélec, FRANCE, frederic.pascal@supelec.fr \\ 3 : French Aerospace Lab, ONERA DEMR/TSI, FRANCE, ovarlez@onera.fr
}

\begin{abstract}
This paper presents a new estimation scheme for optimally deriving clutter parameters with high resolution POLSAR data. The heterogeneous clutter in POLSAR data is described by the Spherically Invariant Random Vectors model. Three parameters are introduced for the high resolution POLSAR data clutter: the span, the normalized texture and the speckle normalized covariance matrix. The asymptotic distribution of the novel span estimator is investigated. A novel heterogeneity test for the POLSAR clutter is also discussed. The proposed method is tested with airborne POLSAR images provided by the ONERA RAMSES system.
\end{abstract}

\section{INTRODUCTION}

The recently launched polarimetric SAR (POLSAR) systems are now capable of producing high quality images of the Earth's surface with meter resolution. The goal of the estimation process is to derive the scene signature from the observed data set. In the case of spatially changing surfaces ("heterogeneous" or "textured" scenes) the first step is to define an appropriate model describing the dependency between the polarimetric signature and the observable as a function of the speckle. In general, the multiplicative model has been employed for POLSAR data processing as a product between the square root of a scalar positive quantity (texture) and the description of an equivalent homogeneous surface (speckle) [1], [2].

This paper is organized as follows. The POLSAR parameter estimation strategy for SIRV clutter model both with normalized texture, and with normalized covariance matrix is presented in Sect. 2 and Sect. 3, respectively. Then, the novel span estimator is introduced in Sect. 4 Next, some estimation results are shown in Sect. 5] on a real high-resolution POLSAR dataset acquired by the ONERA RAMSES system. Eventually, in Sect. 6, some conclusions are presented.

\section{SIRV CLUTTER MODEL WITH NORMALIZED TEXTURE}

The SIRV is a class of non-homogeneous Gaussian processes with random variance [3], [4]. The complex $m$ dimensional measurement $\mathbf{k}$ is defined as the product between the independent complex circular Gaussian vector $\zeta \sim \mathcal{N}(0,[T])$ (speckle) with zero mean and covariance matrix $[T]=E\left\{\zeta \zeta^{\dagger}\right\}$ and the square root of the positive random variable $\xi$ (representing the texture): $\mathbf{k}=\sqrt{\xi} \cdot \zeta$. It is important to notice that in the SIRV definition, the probability density function (PDF) of the texture random variable is not explicitly specified. As a consequence, SIRVs describe a whole class of stochastic processes [5].

For POLSAR clutter, the SIRV product model is the product of two separate random processes operating across two different statistical axes [6]. The polarimetric diversity is modeled by the multidimensional Gaussian kernel. The randomness of spatial variations in the radar backscattering from cell to cell is characterized by $\xi$. Relatively to the polarimetric axis, the texture random variable $\xi$ can be viewed as a unknown deterministic parameter from cell to cell.

The texture and the covariance matrix unknown parameters can be estimated from the ML theory. For $N$ i.i.d. (independent and identically distributed) secondary data, let $L_{\mathbf{k}}\left(\mathbf{k}_{1}, \ldots, \mathbf{k}_{N} \mid[T], \xi_{1}, \ldots, \xi_{N}\right)$ be the likelihood function to maximize with respect to $[T]$ and $\xi_{i}$.

$$
\begin{aligned}
L_{\mathbf{k}}\left(\mathbf{k}_{1}, \ldots, \mathbf{k}_{N} ;[T], \xi_{1}, \ldots, \xi_{N}\right)=\frac{1}{\pi^{m N} \operatorname{det}\{[T]\}^{N}} \times \\
\times \prod_{i=1}^{N} \frac{1}{\xi_{i}^{m}} \exp \left(-\frac{\mathbf{k}_{i}^{\dagger}[T]^{-1} \mathbf{k}_{i}}{\xi_{i}}\right)
\end{aligned}
$$

The corresponding ML estimators are given by [7]:

$$
\begin{aligned}
& \frac{\partial \ln L_{\mathbf{k}}\left(\mathbf{k}_{1}, \ldots, \mathbf{k}_{N} \mid[T], \xi_{1}, \ldots, \xi_{N}\right)}{\partial \xi_{i}}=0 \Leftrightarrow \widehat{\xi}_{i}=\frac{\mathbf{k}_{i}^{\dagger}[T]^{-1} \mathbf{k}_{i}}{m}, \\
& \frac{\partial \ln L_{\mathbf{k}}\left(\mathbf{k}_{1}, \ldots, \mathbf{k}_{N} \mid[T], \xi_{1}, \ldots, \xi_{N}\right)}{\partial[T]}=0 \Leftrightarrow[\widehat{T}]=\frac{1}{N} \sum_{i=1}^{N} \frac{\mathbf{k}_{i} \mathbf{k}_{i}^{\dagger}}{\widehat{\xi}_{i}} .
\end{aligned}
$$


As the variables $\xi_{i}$ are unknown, the following normalization constraint on the texture parameters assures that the ML estimator of the speckle covariance matrix is the Sample Covariance Matrix (SCM):

$[\widehat{T}]=\frac{1}{N} \sum_{i=1}^{N} \mathbf{k}_{i} \mathbf{k}_{i}^{\dagger}=[\widehat{T}]_{S C M} \Leftrightarrow \frac{1}{N} \sum_{i=1}^{N} \mathbf{k}_{i} \mathbf{k}_{i}^{\dagger}\left(1-\frac{1}{\widehat{\xi}_{i}}\right)=\left[0_{m}\right]$.

The generalized ML estimator for $\xi_{i}$ are obtained by introducing Eq. 4 in Eq. 2.

$$
\widehat{\xi}_{i}=\frac{\mathbf{k}_{i}^{\dagger}[\widehat{T}]_{S C M}^{-1} \mathbf{k}_{i}}{m}
$$

Note the $\mathbf{k}_{i}$ primary data is the cell under study.

The normalized texture estimator from Eq. 5 is known as the Polarimetric Whitening Filter (PWF-SCM) introduced by Novak and Burl in [1].

\section{SIRV CLUTTER MODEL WITH NORMALIZED COVARIANCE MATRIX}

Let now the covariance matrix be of the form $[T]=\sigma_{0}[M]$, such that $\operatorname{Tr}\{[M]\}=1$. The product model can be also written as $\mathbf{k}=\sqrt{\tau} \cdot \mathbf{z}$, where $\mathbf{z} \sim \mathcal{N}(\mathbf{0},[M]) . \sigma_{0}$ and $\xi$ are two scalar positive random variables such that $\tau=\sigma_{0} \cdot \xi$.

Using the same procedure as in Sect. 2 and given the fact that the covariance matrix is normalized, it is possible to compute the generalized ML estimator of $[M]$ as the solution of the following recursive equation:

$$
[\widehat{M}]_{F P}=f\left([\widehat{M}]_{F P}\right)=\frac{1}{N} \sum_{i=1}^{N} \frac{\mathbf{k}_{i} \mathbf{k}_{i}^{\dagger}}{\mathbf{k}_{i}^{\dagger}[\widehat{M}]_{F P}^{-1} \mathbf{k}_{i}} .
$$

This approach has been used in [8] by Conte et al. to derive a recursive algorithm for estimating the matrix $[M]$. This algorithm consists in computing the Fixed Point of $f$ using the sequence $\left([M]_{i}\right)_{i \geq 0}$ defined by:

$$
[M]_{i+1}=f\left([M]_{i}\right) .
$$

This study has been completed by the work of Pascal et al. [9], [10], which recently established the existence and the uniqueness, up to a scalar factor, of the Fixed Point estimator of the normalized covariance matrix, as well as the convergence of the recursive algorithm whatever the initialization. The algorithm can therefore be initialized with the identity matrix $[\widehat{M}]_{0}=\left[I_{m}\right]$.

The generalized ML estimator (PWF-FP) for the $\tau_{i}$ texture for the primary data $\mathbf{k}_{i}$ is given by:

$$
\widehat{\tau}_{i}=\frac{\mathbf{k}_{i}^{\dagger}[\widehat{M}]_{F P}^{-1} \mathbf{k}_{i}}{m} .
$$

One can observe that the PWF-FP texture from Eq. 8 has the same form as the PWF-SCM. The only difference is the use of the normalized covariance estimate given by the FP estimator instead of the conventional SCM [6].

\section{MAIN RESULT}

The span (total power) $\sigma_{0}$ can be derived using the covariance matrix estimators presented in Sect. 2 and Sect. 3 as:

$$
\widehat{\sigma_{0}}=\frac{\mathbf{k}^{\dagger}[\widehat{M}]_{F P}^{-1} \mathbf{k}}{\mathbf{k}^{\dagger}[\widehat{T}]_{S C M}^{-1} \mathbf{k}}
$$

Note that Eq. 9 is valid when considering $N$ identically distributed linearly independent secondary data and one primary data. It can be seen as a double polarimetric whitening filter issued from two equivalent SIRV clutter models: with normalized texture variables and with normalized covariance matrix parameter.

The main advantage of the proposed estimation scheme is that it can be directly applied with standard boxcar neighborhoods.

\subsection{Asymptotic statistics of $\widehat{\sigma_{0}}$}

This section is dedicated to the study of large sample properties and approximations of the span estimator $\widehat{\sigma_{0}}$ form Eq. 9

On one hand, the asymptotic distribution of the FP estimator from Eq. 6 has been derived in [10]. The FP estimator computed with $N$ secondary data converges in distribution to the normalized SCM computed with $N[m /(m+1)]$ secondary data. Since the normalized SCM is the SCM up to a scale factor, we may conclude that, in problems invariant with respect to a scale factor on the covariance matrix, the FP estimate is asymptotically equivalent to the SCM computed with $N[m /(m+1)]$ secondary data. Hence one can set the degrees of freedom of FP normalized covariance matrix estimators as:

$$
q_{1}=N \frac{m}{m+1} .
$$

On the other hand, Chatelain et al. established the multisensor bivariate gamma distribution PDF [11]:

$$
P_{b \Gamma}\left(y_{1}, y_{2} ; p_{1}, p_{2}, p_{12}, q_{1}, q_{2}\right) \text {. }
$$

The scale parameters $p_{2}$ and $p_{1}$, the shape parameters $q_{2}>$ $q_{1}$ and $p_{12}$ are linked to the mean parameters $\mu_{1}, \mu_{2}$, to the number of degrees of freedom $n_{1}, n_{2}$, and to the normalized correlation coefficient $\rho$ such as:

$q_{1}=n_{1}, q_{2}=n_{2}, p_{1}=\frac{\mu_{1}}{q_{1}}, p_{2}=\frac{\mu_{2}}{q_{2}}, p_{12}=\frac{\mu_{1} \mu_{2}}{q_{1} q_{2}}(1-\rho)$.

Using these results, we derived the PDF of the ratio $R=$ $y_{1} / y_{2}$ of two correlated Gamma random variables:

$$
\begin{gathered}
P_{R \Gamma}\left(R, p_{1}, p_{2}, p_{12}, q_{1}, q_{2}\right)=R^{q_{1}-1}\left(\frac{p_{2}}{p_{12}}\right)^{q_{1}}\left(\frac{1}{p_{2}}\right)^{q_{2}} \times \\
\times\left(\frac{p_{12}}{p_{1}+R p_{2}}\right)^{q_{2}+q_{1}} \frac{\Gamma\left(q_{1}+q_{2}\right)}{\Gamma\left(q_{1}\right) \Gamma\left(q_{2}\right)} \times
\end{gathered}
$$


$\times \mathbf{H}_{3}\left[q_{1}+q_{2}, q_{2}-q_{1}, q_{2} ; R \frac{p_{1} p_{2}-p_{12}}{\left(p_{1}+R p_{2}\right)^{2}}, \frac{p_{1} p_{2}-p_{12}}{p_{2}\left(p_{1}+R p_{2}\right)}\right]$,

where $\quad \mathbf{H}_{3}(\alpha, \beta, \gamma ; x, y)=\sum_{m, n=0}^{\infty} \frac{(\alpha)_{2 m+n}(\beta)_{n}}{(\gamma)_{m+n} m ! n !} x^{m} y^{n}$ is one of the twenty convergent confluent hypergeometric series of order two (Horn function), and $(\alpha)_{n}$ is the Pochhammer symbol such that $(a)_{0}=1$ and $(a)_{k+1}=(a+k)(a)_{k}$ for any positive integer $k$.

By taking into consideration both Eqs. 10, 11 and the Cochran's theorem, the PDF of the span estimator from Eq. 9 converges asymptotically to the the ratio of two correlated Gamma random variables PDF (the ratio of two quadratics). Moreover, the degrees of freedom $n_{1}$ and $n_{2}$ are set to $N[m /(m+1)]$ and $N$ (the number of secondary data), respectively.

\subsection{The $\sigma_{0}$ test}

In this section we propose to show how the estimator from Eq. 9 is linked with a binary hypothesis testing problem, also:

- under the null hypothesis $H_{0}$, the observed target vector $\mathbf{k}=\sqrt{\xi} \cdot \zeta$ belongs to the SIRV clutter $\zeta \sim \mathcal{N}(0,[T])$ with normalized texture,

- under the alternative hypothesis $H_{1}$, the primary target vector $\mathbf{k}=\sqrt{\tau} \cdot \mathbf{z}$ belongs to the SIRV clutter $\mathbf{z} \sim$ $\mathcal{N}(0,[M])$ with normalized covariance matrix.

The Neyman-Pearson optimal detector is given by the following likelihood ratio test (LRT):

$$
\Lambda(\mathbf{k})=\frac{p_{\mathbf{k}}\left(\mathbf{k} / H_{1}\right)}{p_{\mathbf{k}}\left(\mathbf{k} / H_{0}\right)} \underset{H_{0}}{\stackrel{H_{1}}{\gtrless}} \lambda .
$$

After expressing the PDF under each hypothesis, it results that:

$$
\Lambda(\mathbf{k})=\frac{\frac{1}{\pi^{m} \operatorname{det}\{[M]\} \tau^{m}} \exp \left(-\frac{\mathbf{k}^{\dagger}[M]^{-1} \mathbf{k}}{\tau}\right)}{\frac{1}{\pi^{m} \operatorname{det}\{[T]\} \xi^{m}} \exp \left(-\frac{\mathbf{k}^{\dagger}[T]^{-1} \mathbf{k}}{\xi}\right)} \underset{H_{0}}{\gtrless} \lambda .
$$

By plugging into the LRT the ML texture estimators from Eqs. 5 and 8 we obtain:

$$
\Lambda(\mathbf{k})=\frac{\operatorname{det}\{[T]\}}{\operatorname{det}\{[M]\}}\left(\frac{\mathbf{k}^{\dagger}[T]^{-1} \mathbf{k}}{\mathbf{k}^{\dagger}[M]^{-1} \mathbf{k}}\right)^{m} \underset{H_{0}}{\stackrel{H_{1}}{\gtrless}} \lambda .
$$

Next, we assume the ratio of determinants is a deterministic quantity and we denote it by $\alpha$. This is an approximation, since in practice the ratio of determinants is also computed using the ML estimators of the respective covariance matrix with $N$ secondary data. Finally, by replacing the known covariances by their ML estimates the generalized LRT is:

$$
\Lambda(\mathbf{k})=\alpha \widehat{\sigma_{0}}-m \underset{H_{0}}{\stackrel{H_{1}}{\gtrless}} \lambda .
$$

As $\alpha$ appears as a deterministic quantity only, it is possible to use the PDF derived in Sect. 4.1 to set the decision threshold $\lambda$ for a specific false alarm probability.

\section{RESULTS AND DISCUSSIONS}

The high resolution POLSAR data set, illustrated in Fig. 1 was acquired by the ONERA RAMSES system over Toulouse, France with a mean incidence angle of $50^{\circ}$. It represents a fully polarimetric (monostatic mode) X-band acquisition with a spatial resolution of approximately $50 \mathrm{~cm}$ in range and azimuth. In the upper part of the image one can observe the CNES buildings.

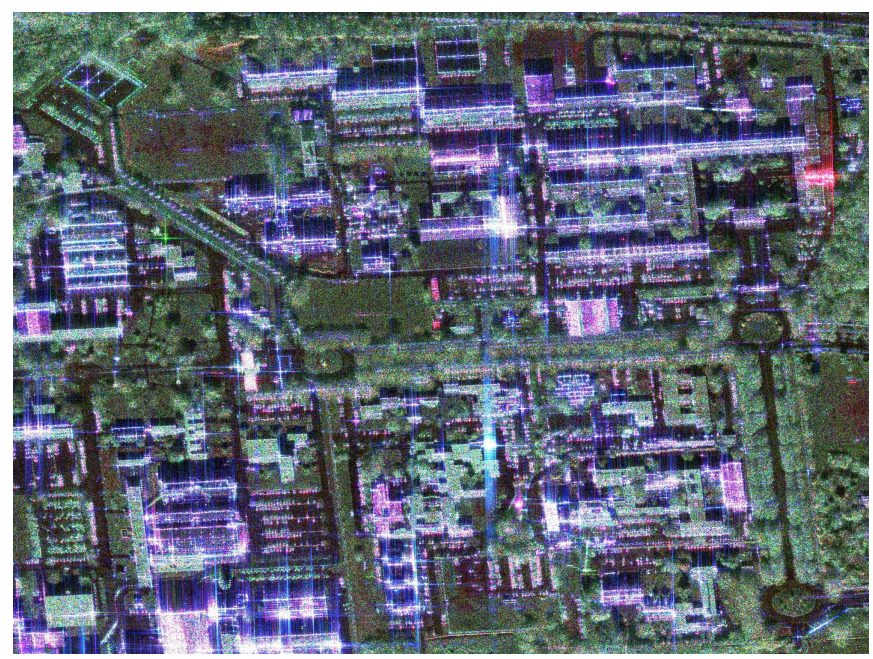

Fig. 1. Toulouse, RAMSES POLSAR data, X-band, $1500 \times$ 2000 pixels: amplitude color composition of the target vector elements $k_{1}-k_{3}-k_{2}$.

Fig. 2 presents the zoom image, where a narrow diplane target was previously detected. Fig. 2-(a),(b),(c) shows the FP-PWF texture, the SCM-PWF normalized texture, and the proposed span estimator $\widehat{\sigma_{0}}$, respectively. For comparison, the Multi-look PWF (MPWF) has been illustrated in Fig. 2 . (d). The proposed estimator exhibits better performances in terms of spatial resolution preservation than the MPWF span estimator: the ring effect (two large dips on a spatial profile near the boundaries of a pointwise target is reduced.

Finally, Fig. 3 illustrates the detection map obtain using the LRT from Eq. 15 with 25 secondary and one primary data. The detection threshold has been obtained by Monte Carlo integration of the PDF from Eq. 11 with a false alarm probability set to $P_{f a}=10^{-3}$ in each pixel. Note that the PDF integration for such a small $P_{f a}$ is quite time consuming and fast numerical approximations need to be investigated in the future for going to an operational level. 


\section{CONCLUSIONS}

This paper presented a new estimation scheme for optimally deriving clutter parameters with high resolution POLSAR images. The heterogeneous clutter in POLSAR data was described by the SIRV model. Three estimators were introduced for describing the high resolution POLSAR data set: the span, the normalized texture and the speckle normalized covariance matrix. The asymptotic distribution of the new span estimator has been established. The estimation bias on homogeneous regions have been assessed also by Monte Carlo simulations. Based on these issues, a novel test has been introduced for selecting the most appropriate model for POLSAR heterogeneous clutter described by SIRVs.

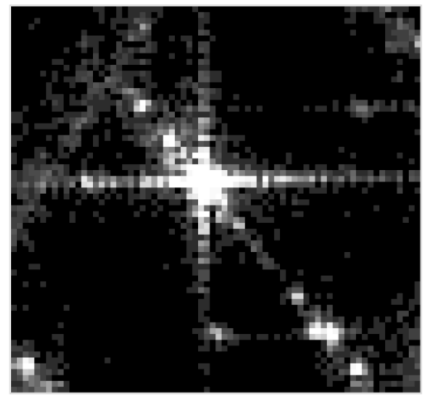

(a)

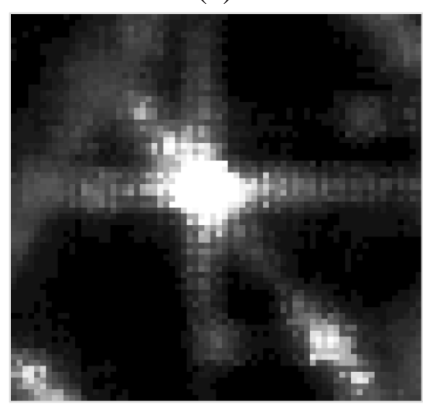

(c)

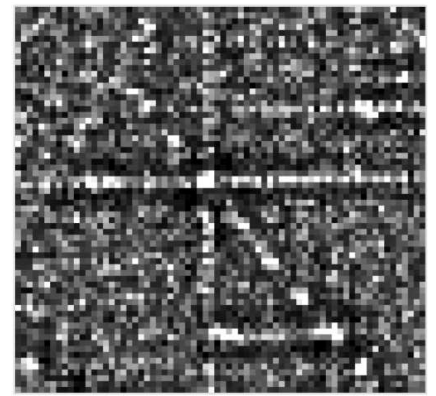

(b)

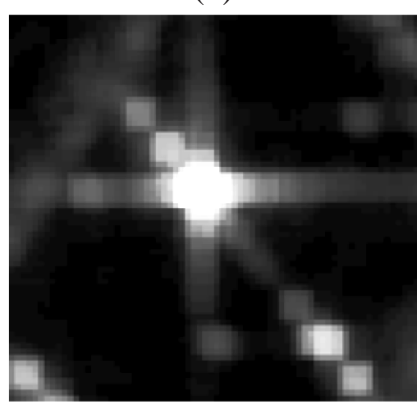

(d)
Fig. 2. Toulouse, RAMSES POLSAR data, X-band, $50 \times$ 50 pixels, zoom image: (a) FP-PWF texture, (b) SCM-PWF normalized texture, (c) span estimated using $\widehat{\sigma_{0}}$ from Eq. 9 and (d) SCM-MPWF span.

\section{REFERENCES}

[1] L. M. Novak and M. C. Burl, "Optimal speckle reduction in polarimetric SAR imagery," IEEE Transactions on Aerospace and Electronic Systems, vol. 26, no. 2, pp. 293-305, 1990.

[2] A. Lopes and F. Sery, "Optimal speckle reduction for the product model in multilook polarimetric SAR imagery and the Wishart distribution," IEEE Transactions on Geoscience and Remote Sensing, vol. 35, no. 3, pp. 632-647, 1997.

[3] B. Picinbono, "Spherically invariant and compound Gaussian stochastic processes," IEEE Transactions on Information Theory, vol. 16, no. 1, pp. 77-79, 1970.

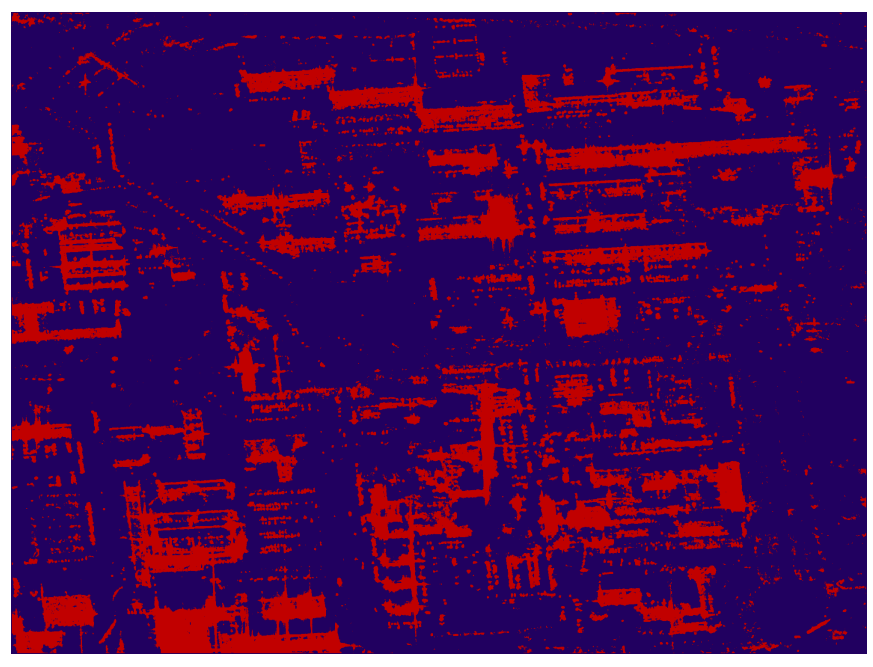

Fig. 3. Toulouse, RAMSES POLSAR data, X-band, $1500 \times$ 2000 pixels: LRT detection map at $P_{f a}=10^{-3}$ (SIRV with normalized texture in blue and SIRV with normalized covariance in red).

[4] K. Yao, "A representation theorem and its applications to spherically-invariant random processes," IEEE Transactions on Information Theory, vol. 19, no. 5, pp. 600-608, 1973.

[5] S. Zozor and C. Vignat, "Some results on the denoising problem in the elliptically distributed context," IEEE Transactions on Signal Processing, vol. 58, no. 1, pp. 134-150, 2010.

[6] G. Vasile, J.-P. Ovarlez, F. Pascal, and C. Tison, "Coherency matrix estimation of heterogeneous clutter in high resolution polarimetric SAR images," IEEE Transactions on Geoscience and Remote Sensing, vol. 48, no. 4, pp. 1809-1826, 2010.

[7] F. Gini and M. V. Greco, "Covariance matrix estimation for CFAR detection in correlated heavy tailed clutter," Signal Processing, vol. 82, no. 12, pp. 1847-1859, 2002.

[8] E. Conte, A. DeMaio, and G. Ricci, "Recursive estimation of the covariance matrix of a compound-Gaussian process and its application to adaptive CFAR detection," IEEE Transactions on Image Processing, vol. 50, no. 8, pp. 1908-1915, 2002.

[9] F. Pascal, Y. Chitour, J.-P. Ovarlez, P. Forster, and P. Larzabal, "Covariance structure maximum-likelihood estimates in compound Gaussian noise: existence and algorithm analysis," IEEE Transactions on Signal Processing, vol. 56, no. 1, pp. 34-48, 2008.

[10] F. Pascal, P. Forster, J.-P. Ovarlez, and P. Larzabal, "Performance analysis of covariance matrix estimates in impulsive noise," IEEE Transactions on Signal Processing, vol. 56, no. 6, pp. 2206-2216, 2008.

[11] F. Chatelain, J. Y. Tourneret, and J. Inglada, "Change detection in multisensor SAR images using bivariate gamma distributions," IEEE Transactions on Image Processing, vol. 17, no. 3, pp. 249-258, 2008. 